\title{
GRAMATICA DE CASOS Y SEMANTICA GENERATIVA (Walter A. Cook, S. J., Georgetown University. Language and Linguistics, Working Papers, Number 8. 1974)
}

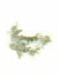

Dice Cook que, antes de comenzar un estudio serio de pragmatismo (pragmatics, el estudio del uso de la lengua) debemos considerar el significado literal de las oraciones. Agrega que, al comparar un sistema como el de la Semántica Generativa que analiza el significado literal en término de estructura lógica (L. S., logical structure) y de estructura de superficie (S.S., surface structure) con el de la Gramática de Casos, en el que la oración se analiza como compuesta de una proposición y una modalidad, son los significados literales los que deben ser comparados, dejando de lado los significados transmitidos.

Con el propósito de establecer los puntos en que ambas gramáticas coinciden y para obtener un beneficio del trabajo de análisis realizado en ambos campos, Cook establece una comparäción entre la Gramática de Casos y la Semántica Generativa considerándolos, primero, desde el punto de vista de sus estructuras profundas, luego, de acuerdo con la forma en que ambas interpretan el análisis causativo y, por último, considerando la manera como ellas analizan la descomposición léxica.

\section{Las estructuras profundas}

Tanto la Gramática de Casos como la Semántica Generativa se interesan en las estructuras profundas que son las que expresan el significado de una oración.

La Semántica Generativa se basa en la teoría de que existe una estructura lógica que subyace en todas las oraciones proposicionales de una lengua y que esta estructura lógica contiene el significado

* Profesora de la Universidad de Costa Rica.
Reseña de Mafalda Bertoglia Richards*

de la oración. La estructura lógica se expresa a través de un predicado central y de una serie de argumentos relacionados con el predicado. El primer paso, en el análisis lógico, es extraer el predicado, el que se escribe en letras mayúsculas para indicar que ya no se considera un ítem léxico sino que un predicado abstracto; el paso siguiente es enumerar los argumentos, colocándolos después del predicado, en el orden de sujeto, objeto indirecto y objeto directo. No se consideran los otros elementos de la oración.

Siguiendo a Cook pero, adaptando sus ejemplos al español, tenemos las siguientes oraciones, con sus respectivos análisis:

(1) Juan es alto. SER ALTO (Juan)

Al convertir las estructuras lógicas en estructuras arbóreas, usaremos los siguientes símbolos: $\mathrm{O}$ (para oración); $\mathrm{F}_{\mathrm{S}} \mathrm{N}_{\mathrm{S}}$ (para frases nominales) y $\mathrm{V}$ (para verbo). El símbolo $\mathrm{O}$ se coloca en el nódulo superior del árbol, el que se ramifica, teniendo en el extremo de una de sus ramas el símbolo $\mathrm{V}$, que representa el predicado y, en las otras ramas, una determinada cantidad de frases nominales (nunca más de tres), que representan los argumentos. La siguiente es la estructura arbórea que representa la oración (1).

\section{(Juan es alto)}

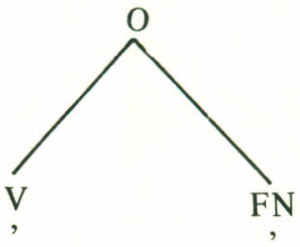

Ser alto

Juan 
Cuando tenemos una oración incrustada dentro de otra oración, debemos reemplazar uno de los nódulos FN del predicado más alto por un nuevo símbolo $\mathrm{O}$ (que denominaremos $\mathrm{O}$ ') y este nuevo símbolo O' domina, a su vez, el predicado más bajo (V) y las $\mathrm{F}_{\mathrm{S}} \mathrm{N}_{\mathrm{S}}$ que están relacionadas con él. Observemos la siguiente oración, y su correspondiente análisis:

(2) Es verdad que Juan es alto. SER VERDAD (SER ALTO (Juan))

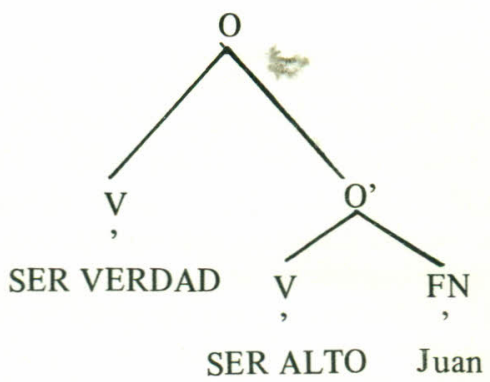

Recordando que en el análisis lógico el primer paso es abstraer el predicado y luego enumerar los argumentos que están directamente relacionados con él, debemos pensar en los diferentes tipos de predicados. Existe el predicado que solamente necesita de un sujeto lógico; es el caso del verbo ser alto, que sólo necesita de un sujeto que tenga la condición de ser alto. Pero también existen predicados que, además de necesitar un sujeto lógico, requieren de un objeto directo y hay otros predicados que necesitan un sujeto lógico, un objeto directo y un objeto indirecto. De estos dos últimos tipos de predicados, matar es un ejemplo del primer caso y dar, uno del segundo.

Considérense los siguientes ejemplos, con sus respectivos análisis:

(3) Juan mató a Guillermo MATAR (Juan, Guillermo)

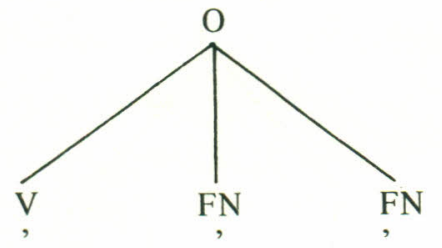

MATAR

Juan

Guillermo
(4) Juan dio un libro a María. DAR (Juan, María, libro)

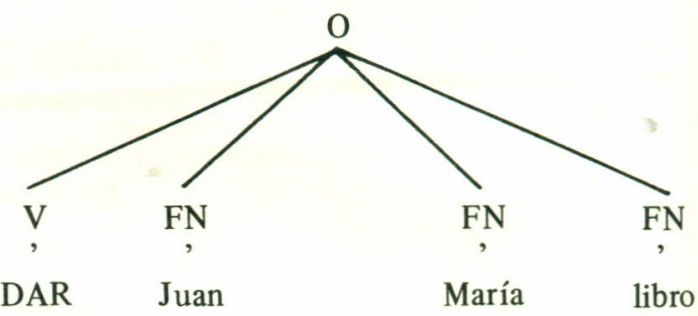

Cook termina su análisis de las estructuras ló. gicas, estableciendo que ellas tienen estructuras muy simples para cada oración, pero árboles muy largos; que la única variedad que se puede obtener es incrustando una oración dentro de otra, ya que sólo hay tres posibles estructuras lógicas que dependen de las exigencias del predicado, y que, de acuerdo con la Semántica Generativa, estas tres posibles estructuras lógicas y combinaciones de ellas se encuentran como las estructuras lógicas que subyacen en todas las posibles oraciones de una lengua.

A diferencia de la Semántica Generativa que describe las estructuras profundas de una oración en términos de estructuras lógicas, la Gramática de Casos lo hace en términos de marcos de casos, difiriendo de aquélla, principalmente en que los argumentos son rotulados como casos y en que los casos son ordenados de izquierda a derecha, de acuerdo con una jerarquía de escogencia del sujeto. Los procedimientos que ambas gramáticas utilizan para determinar las estructuras profundas, son muy semejantes. Observemos la oración siguiente y su correspondiente análisis:

(5) Juan le dio el libro a María. GIVE, + [ A, B, O ]

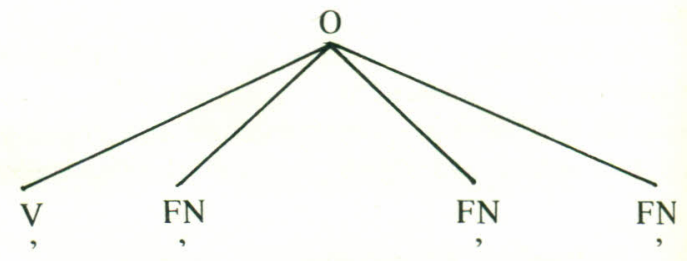

GIVE Juan María libro 
El predicado, GIVE, se abstrae de la oración y se ordena junto con sus argumentos, que se rotulan Agente (A), Beneficiario (B), y Objeto (O). El espacio en blanco, inmediatamente delante de los argumentos y dentro del marco, indica la posición del verbo. Los argumentos se colocan en el orden $\mathrm{A}, \mathrm{B}, \mathrm{O}$, porque A se escoge como sujeto cada vez que aparece; si $\mathrm{A}$ no aparece, la elección recae en B; sólo si A y B no están presentes, $\mathrm{O}$ es escogido como sujeto.

Es interesante recalcar, como lo hace Cook, que mientras menor sea el número de casos en un modelo de Gramática de Casos, más abstracto se hace el modelo y más se acerca a la Semántica Generativa, alejándose de esta última y haciéndose más concreto a medida que más casos se agregan.

El modelo de Gramática de Casos que usa Cook en su estudio se basa en el trabajo de Charles Fillmore, Wallace Chafe y John Anderson y ya había sido desarrollado por el autor en artículos anteriores. Los cinco casos proposicionales son: Agente, Experientivo, Benefactivo, Objeto y Locativo. Cada marco de casos tiene por lo menos un caso y ninguno tiene más de tres. La siguiente es la matriz de los marcos de casos:
Desde un punto de vista, los verbos son clasificados como verbos de Estado, de Proceso o de Acción; desde otro ángulo, se clasifican como Básicôs (acción y objeto, solamente), como Experientivos (sensación, emoción y conocimiento), Benefactivos (posesión, o transferencia de la propiedad) y Locativos (fijación en un lugar, movimiento). Es interesante hacer notar que el caso Objeto aparece en cada uno de los marcos de casos de la matriz y que su significado depende del marco en el cual está. Puede ser el objeto en un estado $\left(\mathrm{O}_{\mathrm{E}}\right)$, puede estar sufriendo un proceso o sentirse afectado por la acción; en otras palabras, es obligatorio. El caso Agente especifica al instigador de la acción; los casos Experientivo, Benefactivo y Locativo se excluyen mutuamente.

Si comparamos ambas gramáticas en lo que respecta a sus estructuras profundas, podemos ver que tanto la Semántica Generativa como la Gramática de Casos, al menos en el estudio de Cook, tienen sólo tres tipos de estructuras profundas, pero que la Gramática de Casos, al rotular los argumentos, tiene la ventaja sobre la Semántica Generativa, de una mayor variedad de estructuras profundas.

\begin{tabular}{lrrrr}
$\begin{array}{l}\text { TIPOS DE } \\
\text { VERBOS }\end{array}$ & VERBOS & EXPERIENTIVOS & BENEFACTIVOS & LOCATIVOS \\
\hline
\end{tabular}
1. De estado
$\mathrm{OE}$
$\mathrm{E}, \mathrm{O}_{\mathrm{E}}$
$\mathrm{B}, \mathrm{OE}$
$\mathrm{OE}_{\mathrm{E}} \mathrm{L}$
ser verdadero
saber
tener
estar en un lugar
2. de proceso
$\stackrel{0}{\text { morir }}$
E, O
$\mathrm{B}, \mathrm{O}$
$\mathrm{O}, \mathrm{L}$
entretener
adquirir
moverse
3. de acción
A, 0
A, E, O
matar
decir
A, B, O
A $\mathrm{O}, \mathrm{L}$
dar
traer 


\section{El análisis causativo}

Este análisis relaciona las formas de estado, las formas incoactivas y las formas causativas del verbo.

El análisis causativo proporciona a la Semántica Generativa, un ejemplo de cómo los predicados complejos que aparecen en las estructuras ló- gicas pueden dividirse en predicados más simples.

Como Cook lo hace notar, varios lingüistas, entre ellos Lakoff, Lyons, Chafe y John Anderson, habían notado el hecho de que algunos verbos tienen formas de estado, formas incoactivas y formas causativas que están morfológicamente relacionadas, y que este hecho constituye un paradigma de significados léxicos relacionados entre sí.

\section{El Paradigma Causativo}

\begin{tabular}{lccc}
\hline Tipo de verbo & Estativo & Incoativo & Causativo \\
\hline Forma léxica & $\begin{array}{c}\text { espeso (a) } \\
\text { adjetivo }\end{array}$ & $\begin{array}{c}\text { espesarse, verbo } \\
\text { intransitivo. }\end{array}$ & $\begin{array}{c}\text { espesar, verbo } \\
\text { transitivo. }\end{array}$ \\
\hline Significado & estar espeso (a) & $\begin{array}{c}\text { llegar a estar } \\
\text { espeso (a) }\end{array}$ & $\begin{array}{c}\text { hacer Hegar a estar } \\
\text { espeso (a) }\end{array}$ \\
\hline
\end{tabular}

El paradigma puede tener fallas léxicas o semánticas, cuando una forma léxica o un significado no existen.

En la Semántica Generativa, se hace uso de dos transformaciones para explicar la relación entre las formas en el paradigma causativo. Se considera la forma estativa como forma base; a ella se le agrega el predicado COME ABOUT (LLEGAR A), para obtener la forma incoativa; a esta última forma se le agrega el predicado CAUSE (HACER) para obtener la forma causativa.

Como un ejemplo de análisis causativo, Cook considera las formas incoativas y causativas que presenta Lakoff.

El predicado estativo, be thick (estar espeso (a)) es la forma base del paradigma; de esta forma se deriva el predicado incoativo, thicken v. i. (espesarse), gracias a la transformación incoativa que agrega el predicado LLEGAR A. Por último, el predicado causativo, thicken v. t. (espesar) se deriva de la forma incoativa que agrega el predicado HACER y un argumento agente.

Observemos los ejemplos y sus representaciones arbóreas:

(6) (La salsa está espesa

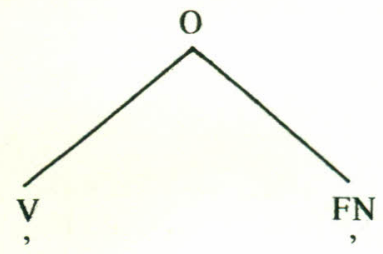

ESTAR ESPESA salsa

(7) (La salsa se espesa)

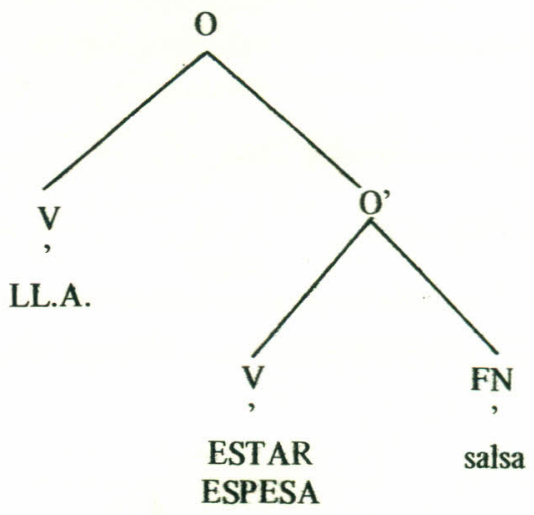


(8) (Max espesa la salsa)

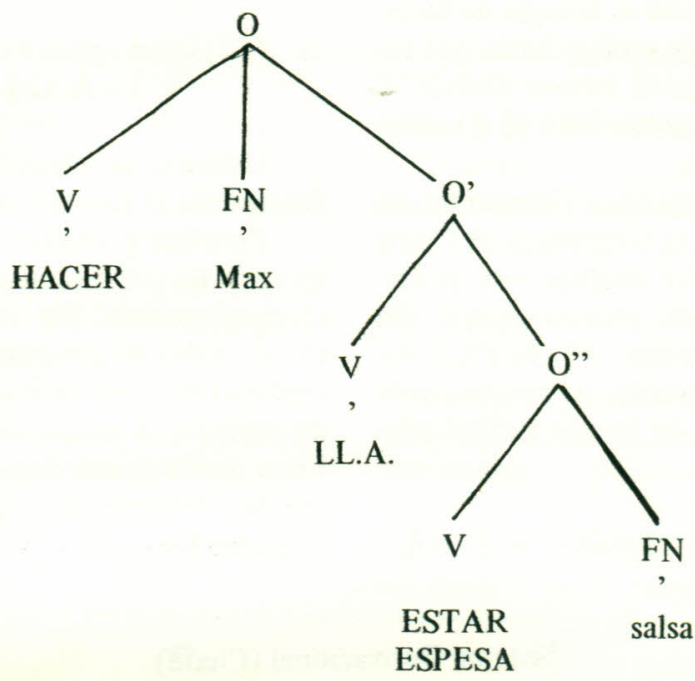

(Nota: LL.A. por LLEGAR A)

Para derivar estructuras superficiales de estructuras profundas, necesitamos de un grupo de reglas que unan las etapas de la derivación. Las estructuras lógicas necesitan de, por lo menos, una regla de formación del sujeto, que invierta el verbo y su sujeto:

(9)

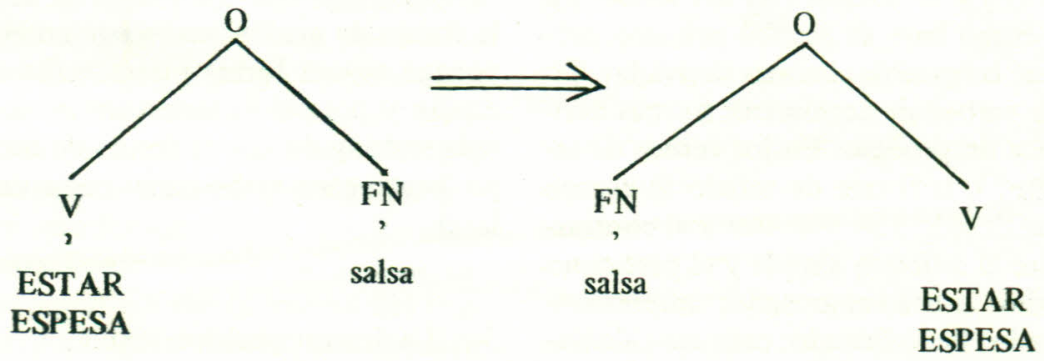


Cuando existe más de un predicado, el predicado más bajo se levanta y se une al mismo nódulo $\mathrm{V}$ del predicado más alto. Esta es la regla de elevación del predicado y se puede aplicar hasta que todos los predicados estén bajo el mismo nódulo V.

Las frases nominales quedan bajo el dominio directo de $\mathrm{O}$.

Contrastando con la Semántica Generativa, en relación al análisis causativo, la Gramática de Casos tiene la ventaja de que dicho análisis está contenido en la dimensión estado-proceso-acción del modelo matriz de su gramática. Al clasificar los predicados como verbos de estado, de proceso o de acción, surge un paradigma de ítemes léxicos relacionados entre sí:

(10)El camino es ancho. Estado, + [ $-\mathrm{OE}]$
(11)El camino se ensancha. Proceso, $+[-\mathrm{O}]$

(12) Juan ensancha el camino. Acción, $+[-\mathrm{A}, \mathrm{O}]$

Después de clasificar los predicados, se debe determinar la forma base del verbo.

Para los gramáticos de casos, las derivaciones incoativas y causativas trabajan también, en la dirección opuesta. Un verbo de acción se convierte en un verbo de proceso por medio de la derivación decausativa y un verbo de proceso llega a ser verbo de estado, a causa de la derivación resultativa. Estas derivaciones demuestran cómo los verbos de estado, de proceso y de acción pueden estar relacionados entre sí.

\section{Sistema Derivacional (Chafe)}
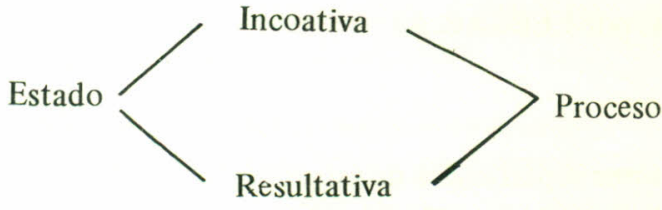

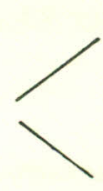

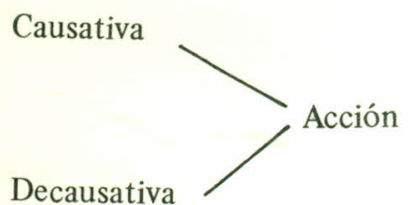

El sistema de la Semántica Generativa es unidireccional, en lo que al análisis causativo se refiere, y supone que la forma de estado del verbo es la forma base, mientras que la Gramática de Casos, en el mismo análisis, usa un sistema bidireccional y tiene que determinar la forma base.

Los verbos tipos se basan en formas de estado (E), de proceso (P) y de acción (A) del verbo. La forma escogida como base se escribe primero, luego, en paréntesis, las posibles formas derivadas. Tipo $\mathrm{A}(\mathrm{E})(\mathrm{P})=$ verbos de acción con formas derivadas de estado y de proceso. En los verbos de estado $\left(+\left[-\mathrm{O}_{\mathrm{E}}\right]\right)$, la forma de estado se escoge como forma base debido a su sencillez y al contraste existente entre el adjetivo simple y el participio pasado del verbo: grueso-engrosado; amplio-ampliado; seco-secado; lleno-llenado; caliente-calentado.

En los verbos de proceso $\left(+\left[-\mathrm{O}_{\mathrm{E}}\right]\right)$, se escoge, como la forma base, la forma de proceso, considerando que es más sencilla, si se le compara con la forma de estado, y que tiene un uso sintáctico libre al compararla con la forma de acción del verbo. La forma de proceso en los verbos de proceso es un verdadero intransitivo:

Tipo P (E) (A) - roto (a), adjetivo (E); romperse, verbo intransitivo $(\mathrm{P})$; romper, verbo transitivo (A).

Al considerar los verbos de acción, vemos que la forma de acción, se escoge como la forma base, porque es una forma más sencilla que la forma de estado y porque la forma de proceso tiene un uso más restringido que la forma de acción. A este grupo, pertenecen verbos tales como cortar, levantar y lavar.

\section{La descomposición léxica.}

Tanto la Semántica Generativa como la Gramática de Casos están interesadas en la descomposición léxica de los predicados abstractos. 
TIPOS DE VERBOS BASADOS EN EL PARADIGMA CAUSATIVO

\begin{tabular}{|c|c|c|c|}
\hline Tipo de verbo & $\begin{array}{l}\text { Forma de } \\
\text { Estado }\end{array}$ & $\begin{array}{c}\text { Forma de } \\
\text { Proceso }\end{array}$ & $\begin{array}{c}\text { Forma de } \\
\text { Acción }\end{array}$ \\
\hline $\mathrm{E}$ & pesado & $*$ & $*$ \\
\hline $\mathrm{E}(\mathrm{P})$ & $\begin{array}{l}\text { no hay } \\
\text { ejemplos }\end{array}$ & & \\
\hline $\mathrm{E}(\mathrm{A})$ & rico & * & enriquecer \\
\hline $\mathrm{E}(\mathrm{P})(\mathrm{A})$ & abierto (adj) & abrirse & abrir \\
\hline $\mathrm{P}$ & $* *$ & estornudar & $*$ \\
\hline$P(E)$ & muerto & morir & * \\
\hline$P(A)$ & $* *$ & golpearse & golpear \\
\hline$P(E)(A)$ & roto & romperse & romper \\
\hline A & $* *$ & $* *$ & reírse \\
\hline$A(E)$ & comido & $* *$ & comer \\
\hline $\mathrm{A}(\mathrm{P})$ & & & no hay ejemplo \\
\hline$A(E)(P)$ & cortado & cortarse & cortar \\
\hline
\end{tabular}

(Nota: * - falla léxica

** - falla semántica)

Para determinar el significado de una oración, la Semántica Generativa abstrae el predicado central y coloca en lista los argumentos que ese predicado necesita. Luego el predicado abstracto se examina para comprobar si puede descomponerse en predicados más simples. La idea es reducir todos los predicados a un grupo de predicados atómicos que ya no se puedan descomponer. Para cumplir con este objetivo, se sugieren posibles construcciones léxicas que se verifican por medio de argumentos semánticos y sintácticos. El análisis de McCawley hace del verbo MATAR es un buen ejemplo de este tipo de análisis. Los vacíos léxicos del paradigma causativo sugieren que algunas formas que no están morfológicamente relacionadas, podrían usarse para llenar esos vacíos. Lakoff sugiere que el verbo matar (causar la muerte de) puede completar el paradigma muerto-morir-matar. McCawley, entonces, analiza la estructura profunda del verbo y usa el análisis como un argumento que demuestra la existencia de transformaciones preléxicas:
(13)Juan mató a Enrique HACER (Juan, LLEGAR A ( ESTAR MUERTO (ENRIQUE))

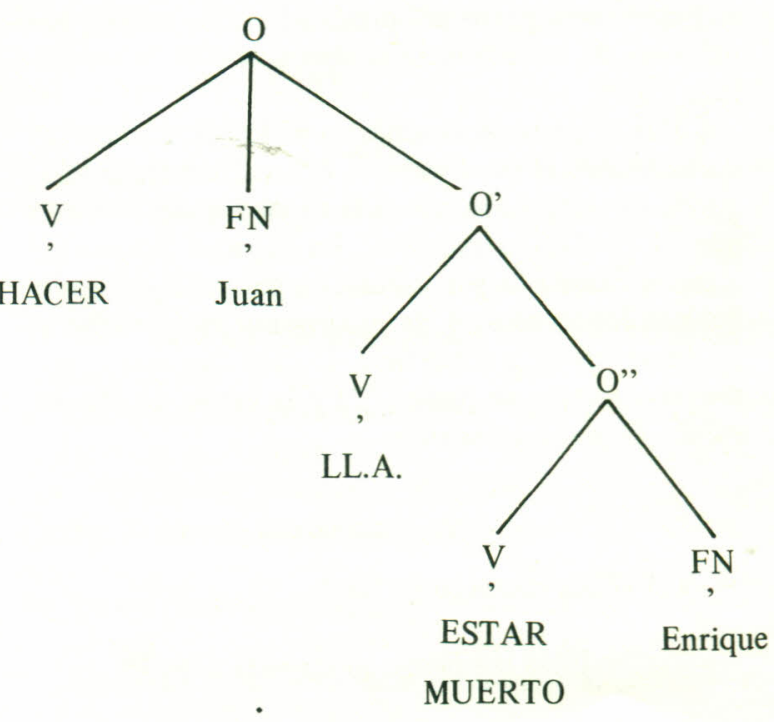


Para justificar su análisis, McCawley señala que el uso del adverbio casi, hace que la oración sea ambigua y que pueda considerarse desde tres diferentes puntos de vista, de acuerdo con la parte del predicado que dicho adverbio modifica directamente en la estructura profunda de la oración

(14)Juan casi mató a Enrique.

(15)Juan casi hizo que Enrique muriera (Juan realmente no hizo nada)

= casi, HACER, LLEGAR A, ESTAR MUERTO

(16)Juan hizo que casi se produjera la muerte de Enrique

(Juan estuvo muy cerca de acabar con su víctima).

$=$ HACER, casi, LLEGAR A, ESTAR MUERTO

(17)Juan hizo que se produjera el que Enrique casi muriera.

(en este caso la víctima queda en un estado cercano a la muerte).

McCawley hace uso del argumento 'modificación' que establece que si un adverbio modifica parte de un predicado, y no el preciado completo, entonces esta parte del predicado debe existir como uno de los predicados abstractos de la estructura lógica.

Por su parte, la Gramática de Casos, aún considerando qủe el predicado es central, centra la búsqueda de estrcturas semánticas en los argumentos que el predicado requiere. En algunas ocasiones, algunos casos que son esenciales para el significado del predicado central, se incorporan en el verbo y no aparecen en la superficie. El caso que está totalmente cubierto se marca (*) y la razón de ello se coloca después del marco.

Ejemplo:

(18) Ellos comieron a las 7.

$=$ Ellos comieron su comida a las 7 .

$$
+\left[-\mathrm{A}, \mathrm{O}^{*}\right] / \mathrm{O}-\mathrm{lex} .
$$

Existen también algunos verbos que llevan incorporado el caso Locativo.

Ejemplo:

(19)Juan se quitó su sombrero.

$=$ Se lo quitó de la cabeza.

$+\left[-\mathrm{A}, \mathrm{O}, \mathrm{L}^{*}\right] /$ L-Lex.

Existe, además, la posibilidad de que algunos casos que son esenciales en el significado del predicado central, estén totalmente cubiertos, porque son correferenciales con algún otro caso.

Ejemplos:

(20) La carta fue a Limón $+[-\mathrm{O}, \mathrm{L}]$

Juan fue a limón $+\left[-\mathrm{A}, \mathrm{O}^{*}, \mathrm{~L}\right] / \mathrm{A}=\mathrm{O}$

(21) El ruido asustó al bebé $+[-\mathrm{O}, \mathrm{E}]$

Juan asustó al bebé $+\left[-\mathrm{A}, \mathrm{E}, \mathrm{O}^{*}\right]$ / A $=\mathrm{O}$

Al comparar la Gramática de Casos con la Semántica Generativa, se han podido comprobar las similitudes que existen entre ambas y el hecho de que difieren, principalmente, en el uso de argumentos rotulados y argumentos no rotulados.

En relación a la estructura semántica, los gramáticos de casos podrían mejorar su análisis, incorporando las estructuras lógicas que usan los semánticos generativistas. Por su parte, los semánticos generativistas podrían considerar la naturaleza bidireccional del proceso de derivación en el análisis causativo.

En lo que respecta a las construcciones léxicas hipotéticas (descomposición léxica), los gramáticos de casos podrían obtener provecho del argumento modificación y, por su parte, los semánticos generativistas podrían considerar los tipos de incorporación léxica, descubiertos por los gramáticos de casos.

Cook termina su estudio estableciendo que todo lo que en él se ha dicho se refiere a la proposición y que no se ha considerado la modalidad. Todavía no existe la posibilidad de que, al analizar una oración, se pueda delinear su total estructura profunda. 